\title{
Approximate solution for high-order fractional integro-differential equations via trigonometric basic functions
}

\author{
B AGHELI* and M ADABITABAR FIROZJA \\ Department of Mathematics, Qaemshahr Branch, Islamic Azad University, Qaemshahr, Iran \\ e-mail: b.agheli@yahoo.com
}

MS received 5 February 2018; revised 23 October 2018; accepted 24 October 2018; published online 14 March 2019

\begin{abstract}
An approach for searching an approximate solution of high-order integro-differential equations featuring fractional derivatives has been proposed in this paper. Initially, using trigonometric basic functions (TBFs), we determine the transformation functions in association to TBFs. Next, the approximate function is presented as a combination of TBFs and transform functions. The convergence of this approach is also presented. Using discrete derivatives of the solution to gain an approximate solution, we find the approximate solution, which satisfies the high-order integro-differential equations featuring fractional derivatives. An algorithm of this approach is applied for various examples, and one example is illustrated in detail.
\end{abstract}

Keywords. High-order fractional integro-differential equations; trigonometric transform; fractional derivative.

\section{Introduction}

Calculus and differential equations of non-integer order (or fractional differential equations (FDEs)) have many utilizations in the real world in different branches of sciences and topics of engineering. Some of these applications were offered by Sun and his associates in [1]. These topics may be included in sciences such as physics, biology, environmental science and topics of engineering such as control, signal processing, image processing, mechanics and dynamic systems.

We invite interested readers to check good books that have been written to get a better grasp of calculus with noninteger derivatives and non-integer integrals [2-4].

Consider fractional integro-differential equations (FIDEs) of the form

$$
\begin{gathered}
D^{\gamma} u(\tau)+\eta \int_{0}^{\tau} K(s, \tau) H[u(s)] d s=g(\tau), \\
M-1<\gamma \leq M, 0<\tau<b, M \in Z^{+} \\
u(0)=u_{0}, \quad u^{(M)}(0)=\theta_{M}
\end{gathered}
$$

where $K(s, \tau)$ and $g(\tau)$ are given, $H[u(s)]$ is any linear function, $u_{0}$ and $\theta_{M}$ are real constants and $D^{\gamma}$ signifies the Caputo derivative of non-integer order $\gamma$ given by

$$
\begin{aligned}
D^{\gamma} u(\tau) & =\frac{1}{\Gamma(m-\gamma)} \int_{0}^{\tau}(\tau-s)^{\gamma-1} u^{(M)}(s) d s, \\
M-1<\gamma & \leq L, \quad M \in Z^{+} .
\end{aligned}
$$

We shown that by the trigonometric transforms method (TTM), it is possible to get an approximate solution for these FIDEs.

Some kinds of FIDEs are found in mathematical modelling of various real-life phenomena such as in

- Physics

About these phenomena, a diversity of physical problems have been formulated as FIDEs. This kind of equation appears in the radiative equilibrium [5]. One of the different requirements for thermodynamic equilibrium is radiative equilibrium.

Heat conduction in materials featuring memory is one example where these equations appear in the mathematical modelling of different physical phenomena [6].

- Ecology

Population dynamics is a branch of ecology that studies the size and age composition of populations as dynamical systems. A population model is a type of mathematical model of FIDE that is utilized in the study of population dynamics $[7,8]$.

- Control

The topic of optimal control is devoted to the problem of finding a control rule for the given system in such a way as to achieve a certain optimal criterion. The fractional optimal control problems (FOCPs) are optimal control problems (OCPs) where differential equations or implement indicator or both feature derivatives

*For correspondence 
of non-integer order. A formulation to FOCPs with derivatives of non-integer order and a solution were introduced in [9]. Rabiei et al [10] presented a approach to solve the FOCP described by FIDEs.

Due to the difficulty and impossibility in obtaining precise solutions for a lot of FDEs and FIDEs, some researchers and scholars use numerical or approximate solution approaches to get the solution to these problems. Among the approximate approaches used by some researchers for FDEs and FIDEs are homotopic analysis (HAM) and optimum $q$-homotopic analysis method (Oq-HAM) $[11,12]$, optimal homotopic perturbation (OHPM) and homotopic perturbation method (HPM) [13, 14], Adomian's decomposition method (ADM) [15], variational iteration method (VIM) [13, 15] and collocation approach $[16,17]$, etc. [18, 19].

Perfilieva [20] was able to gain a solution for ordinary differential equations by the trigonometric transforms. In $[21,22]$ Khastan used the TTM to get an approximate solution for fuzzy differential equations of first order. Tomasiello [23] used the TTM to gain an approximate solution for delay differential equations.

Chen and Shen [24] have utilized an algorithm to get the approximate solutions of initial value problems (IVPs) of second order.

\section{Preliminaries}

In this section, two subsections have been considered. In the first subsection, discretization of the fractional derivative for different orders is presented. In the second subsection, we present the TBFs, lemmas and theorems to approximate the function of the solution, including convergence of approximate solution function with TBFs.

\subsection{Discretization of the derivative of non-integer order}

The discretization of derivative of non-integer order is introduced in this subsection. It is possible that by forward differences in finite difference, we gain the approximation of derivatives. Especially, for IVPs it is possible. The $k$-th order forward difference is given by

$$
x^{(k)}(t) \approx \frac{1}{L^{k}} \sum_{p=0}^{k}\left(\begin{array}{l}
k \\
p
\end{array}\right)(-1)^{p} x((k-p) h+t), \quad k \in N,
$$

in which $L$ may be constant or variable pertaining to the application. We let $L=s_{j+1}-s_{j}$ and $s_{j}=a+j L$, $j=0,1,2,3, \ldots$, for this paper.

Li et al [25] obtain the approximation concerning the Caputo derivative. For Eq. (3), regarding the approximation concerning the Caputo derivative and $0<\gamma \leq 1$

$$
\begin{aligned}
D^{\gamma} u\left(s_{k+1}\right) \approx & \frac{1}{L^{\gamma} \Gamma(2-\gamma)} \sum_{j=0}^{k}\left(u\left(s_{j+1}\right)-u\left(s_{j}\right)\right) \\
& \times\left((k-j+1)^{1-\gamma}-(k-j)^{1-\gamma}\right),
\end{aligned}
$$

in which $u\left(s_{0}\right)$ is known and for $1<\gamma \leq 2$

$$
\begin{aligned}
D^{\gamma} u\left(s_{k+1}\right) \approx & \frac{1}{L^{\gamma} \Gamma(3-\gamma)} \sum_{j=0}^{k}\left(u\left(s_{j+1}\right)-2 u\left(s_{j}\right)+u\left(s_{j-1}\right)\right) \\
& \times\left((k-j+1)^{2-\gamma}-(k-j)^{2-\gamma}\right),
\end{aligned}
$$

in which $u\left(s_{-1}\right)=u(0)-L \frac{d}{d t} u(0), u\left(s_{0}\right)$ and $\frac{d}{d t} u\left(s_{0}\right)$ are known and for $2<\gamma \leq 3$

$$
\begin{aligned}
D^{\gamma} u\left(s_{k+1}\right) \approx & \frac{1}{L^{\gamma} \Gamma(4-\gamma)} \sum_{j=0}^{k}\left(u\left(s_{j+2}\right)-3 u\left(s_{j+1}\right)\right. \\
& \left.+3 u\left(s_{j}\right)-u\left(s_{j-1}\right)\right) \\
& \times\left((k-j+1)^{3-\gamma}-(k-j)^{3-\gamma}\right),
\end{aligned}
$$

in which $u\left(s_{1}\right)=\frac{d}{d t} u\left(s_{0}\right)-L \frac{d^{2}}{d t^{2}} u\left(s_{0}\right), u\left(s_{0}\right), \frac{d}{d t} u\left(s_{0}\right)$ and $\frac{d^{2}}{d t^{2}} u\left(s_{0}\right)$ are known.

\subsection{Trigonometric basic functions (TBFs)}

We present the trigonometric basic functions (TBFs) and properties that are applied in the new approach to the approximate solution, in this subsection.

Definition 1 Consider nodes $a=s_{0}<s_{1}<\cdots<s_{n-1}<$ $s_{n}=b$ for $n \geq 1$. The basic functions (BFs)

$$
S_{0}, S_{1}, \ldots, S_{n}
$$

on interval $[a, b]$ are defined with trigonometric functions (TFs) $S_{0}, S_{1}(t), \ldots, S_{n}(t)$, as follows, where $h_{p}=s_{p+1}-s_{p}$ and $p=0,1,2, \ldots, n-1$ :

$$
\begin{aligned}
& S_{0}(t)=\left\{\begin{array}{cc}
0.5\left(1+\cos \frac{\left(t-s_{0}\right)}{h_{0}} \pi\right), & s_{0} \leq t \leq s_{1} \\
0, & \text { otherwise },
\end{array}\right. \\
& S_{k}(t)=\left\{\begin{array}{cc}
0.5\left(1+\cos \frac{\left(t-s_{k}\right)}{h_{k-1}} \pi\right), & s_{k-1} \leq t \leq s_{k}, \\
0.5\left(1+\cos \frac{\left(t-s_{k}\right)}{h_{k}} \pi\right), & s_{k} \leq t \leq s_{k+1}, \\
0, & \text { otherwise }
\end{array}\right. \\
& S_{n}(t)=\left\{\begin{array}{cc}
0.5\left(1+\cos \frac{\left(t-s_{n}\right)}{h_{n-1}} \pi\right), & s_{n-1} \leq t \leq s_{n} \\
0, & \text { otherwise },
\end{array}\right.
\end{aligned}
$$

in which $k=2,3,4, \ldots, n-1$. 
Consider the TFs in Definition 1. They are TBFs whenever the following properties are satisfied:

1) Continuity for $S_{k}$ of $[a, b]$ to $[0,1]$,

2) $\sum_{k=0}^{n} S_{k}(t)=1, t \in[a, b]$,

3) $S_{k}\left(s_{k}\right)=1$, for $k=0,1,2,3, \ldots, n$,

4) $S_{k}(t)=0$, if $t \notin\left(s_{k-1}, s_{k+1}\right)$, for $k=1,2, \ldots, n-1$,

5) $S_{0}(t)=0$, if $t \notin\left(s_{0}, s_{1}\right)$,

6) $S_{n}(t)=0$, if $t \notin\left(s_{n-1}, s_{n}\right)$,

7) on interval $\left[s_{k-1}, s_{k+1}\right], S_{k}(t)$, increases function on $\left[s_{k-1}, s_{k}\right], k=1,2,3, \ldots, n-1$,

8) on interval $\left[s_{k-1}, s_{k+1}\right], S_{k}(t)$, decreases function on $\left[s_{k}, s_{k+1}\right], k=1,2,3, \ldots, n-1$,

9) $S_{k}\left(t+s_{k}\right)=S_{k}\left(s_{k}-t\right), t \in[0, h], k=1,2, \ldots, n-1$,

10) $S_{k}(t)=S_{k-1}(t-h), S_{k+1}(t)=S_{k}(t-h), k=1,2, \ldots$, $n-1, t \in\left[s_{k}, s_{k+1}\right]$.

BFs are named uniform functions with regard to $s_{k+1}-$ $s_{k}=h=\frac{b-a}{n}$ and properties (9) and (10) coincide.

Lemma 1 [20] Consider $n \geq 2, S_{0}, S_{1}, \ldots, S_{n}$, are TBFs defined on $[a, b]$. Then we have

$$
\int_{s_{k-1}}^{s_{k+1}} S_{k}(t) d t=h, \quad k=1,2,3, \ldots, n-1 .
$$

The interval between two neighbouring nodes is assumed be $h$, and

$$
\int_{s_{0}}^{s_{1}} S_{1}(t) d t=\int_{s_{n-1}}^{s_{n}} S_{n}(t) d t=\frac{h}{2} .
$$

Lemma 2 Suppose sinusoidal membership functions for $n \geq 2, S_{0}, S_{1}, S_{2}, \ldots, S_{n}$, be the TBFs defined on $[a, b]$. Thus for $k=0,1,2,3, \ldots, n$

$$
\int_{0}^{s_{k}} S_{j}(t) d t=\left\{\begin{array}{lc}
h, & k \geq j+1, \\
0, & k \leq j-1, \\
\frac{h}{2}, & k=j .
\end{array}\right.
$$

Proof When $t \notin\left(s_{j-1}, s_{j+1}\right)$, from Definition $1, S_{j}(t)=0$. When $k \geq j+1$, from Definition 1 and Lemma 1 , the result will be

$$
\int_{0}^{s_{k}} S_{j}(t) d t=\int_{0}^{s_{k-1}} S_{j}(t) d t+\int_{s_{k-1}}^{s_{k+1}} S_{j}(t) d t+\int_{s_{k+1}}^{s_{k}} S_{j}(t) d t=h .
$$

When $k \leq j-1, t \leq s_{k}$; hence, $S_{j}(t)=0$ and we get $\int_{0}^{s_{k}} S_{j}(t) d t=0$.

When $k=j$, from Lemma 2 and Definition 1

$$
\int_{0}^{s_{k}} S_{j}(t) d t=\int_{0}^{s_{k-1}} S_{j}(t) d t+\int_{s_{k-1}}^{s_{k}} S_{j}(t) d t=\frac{h}{2} .
$$

Definition 2 Consider intervals $H=C([a, b]), I=[a, b]$. Let $f \in H$ and $S_{j}, j=0,1, \ldots, n$, be the TBFs defined on
I. We assume that $P_{j}$ is the transform of function $p$ on $I$; considering BFs $S_{j}, j=0,1,2,3, \ldots, n$, we get

$$
P_{j}=\frac{\int_{a}^{b} f(t) S_{j}(t) d t}{\int_{a}^{b} S_{j}(t) d t} .
$$

Definition 3 Consider intervals $H=C([a, b]), I=[a, b]$. Let $f \in H$ and $S_{i}, i=0,1, \ldots, n$, be the TBFs defined on $I$ and $P_{k}$ be the transform of function $p$ on $I$ considering BFs $S_{k}$. Then

$$
P_{n}(t)=\sum_{k=0}^{n} P_{k} S_{k}(t)
$$

is an approximation of function $p$ on $I$, considering TBFs. For convergence, we have the following theorem.

Theorem 1 Suppose $h$ is a monotonic continuous function on $I=[a, b]$. For all $\epsilon>0$, there exists $n_{\epsilon}$ such that for any $n \geq n_{\epsilon}$

$$
\left|h(t)-h_{n_{\epsilon}}(t)\right|<\epsilon
$$

Proof For $0<\delta<\epsilon$, we have $\forall \epsilon>0, \quad \exists \delta=\delta(\epsilon),|x-t|$ $<\delta \Rightarrow|h(x)-h(t)|<\epsilon$, because $h$ is a monotonic continuous function on $I$.

When $\epsilon>0$

$$
\begin{aligned}
\left|h(t)-h_{n}(t)\right| & =\left|\sum_{i=0}^{n} S_{i}(t) h(t)-\sum_{i=0}^{n} P_{i} S_{i}(t)\right| \\
& \leq \sum_{i=0}^{n} S_{i}(t)\left|h(t)-P_{i}\right|<\epsilon .
\end{aligned}
$$

This is sufficient to show that $\left|h(t)-P_{i}\right|<\epsilon$. Assume $t, x \in\left[x_{i-1}, x_{i+1}\right], i=1,2, \ldots, n-1$. Then

$$
\begin{aligned}
\left|h(x)-P_{i}\right| & =\left|h(x)-\frac{\int_{a}^{b} h(t) S_{i}(t) d t}{\int_{a}^{b} S_{i}(t) d t}\right| \\
& \leq \frac{\int_{x_{i-1}}^{x_{i+1}} S_{i}(t)|h(x)-h(t)| d t}{\int_{x_{i-1}}^{x_{i+1}} S_{i}(t) d t}<\epsilon
\end{aligned}
$$

iff $\delta<2 \mathrm{~h}<\epsilon$.

Thus, $n_{\epsilon}>\frac{2(b-a)}{\epsilon}$ is suitable; assume $h=\frac{b-a}{n}$.

Proposition 1 Replace $p_{n}(t)=\sum_{k=0}^{n} P_{k} S_{k}(t)$ in Eqs. (4)(6); then for $k=0,1,2,3, \ldots, n-1$

$$
\begin{aligned}
D^{\gamma} p_{n}\left(s_{k+1}\right) \approx & \frac{1}{L^{\gamma} \Gamma(2-\gamma)} \sum_{j=0}^{k}\left(P_{j+1}-P_{j}\right) \\
& \times\left((k-j+1)^{1-\gamma}-(k-j)^{1-\gamma}\right), \\
& 0<\gamma \leq 1 .
\end{aligned}
$$




$$
\begin{aligned}
D^{\gamma} p_{n}\left(s_{k+1}\right) \approx & \frac{1}{L^{\gamma} \Gamma(3-\gamma)} \sum_{j=0}^{k}\left(P_{j+1}-2 P_{j}+P_{j-1}\right) \\
& \times\left((k-j+1)^{2-\gamma}-(k-j)^{2-\gamma}\right), \\
& 1<\gamma \leq 2 . \\
D^{\gamma} p_{n}\left(s_{k+1}\right) \approx & \frac{1}{L^{\gamma} \Gamma(4-\gamma)} \sum_{j=0}^{k}\left(P_{j+2}-3 P_{j+1}+3 P_{j}-P_{j-1}\right) \\
& \times\left((k-j+1)^{3-\gamma}-(k-j)^{3-\gamma}\right), \\
& 2<\gamma \leq 3 .
\end{aligned}
$$

\section{Description of the new approach}

Assume the solution of Eq. (1) to be continuous on $I_{1}=[0, b]$. To obtain an approximate solution of $u(x)$, divide $I_{1}$ to $n$ equal partitions involving step length $L$, for $i=0,1, \ldots, n$ :

$$
s_{0}=0, \quad s_{i}=s_{0}+i L, \quad L=\frac{b}{n} .
$$

Considering the TFs with Definition 1 on $I_{1}$ and Definition 3, we get an approximation of function $u(x) \quad$ as $\quad u_{n}(x)=\sum_{k=0}^{n} U_{k} S_{k}(t)$. Thus, to calculate $u_{n}(t)$, we should calculate $U_{k}, k=0,1, \ldots, n$, when $t \in[0, b]$. To get an approximate solution of Eq. (1), $u_{n}(t)$ for points $s_{0}, s_{1}, \ldots, s_{n}$ must satisfy Eq. (1). Due to the boundary conditions Eq. (2), $u_{n}\left(s_{0}\right):=u\left(s_{0}\right)=u_{0}$ and for other points $s_{1}, s_{2}, \ldots, s_{n}, m-1<\gamma \leq m$, we have

$$
\begin{array}{r}
D_{t}^{\gamma} u_{n}\left(s_{k+1}\right)+\eta \int_{0}^{s_{k+1}} K\left(s_{k+1}, s\right) u_{n}(s) d s=g\left(s_{k+1}\right), \\
k=0,1,2,3, \ldots, n-1,
\end{array}
$$

where $m \in Z^{+}$.

Regarding Caputo's derivative, using Eqs. (12)-(14), Eq. (16) is converted to the following form for $k=0,1,2, \ldots, n-1$ :

$$
\begin{aligned}
& \frac{1}{L^{\gamma} \Gamma(2-\gamma)} \sum_{j=0}^{k}\left(U_{j+1}-U_{j}\right) \\
& \quad \times\left((k-j+1)^{1-\gamma}-(k-j)^{1-\gamma}\right) \\
& \quad+\eta \int_{0}^{s_{k+1}} K\left(s_{k+1}, s\right) u_{n}(s) d s=g\left(s_{k+1}\right), \\
& 0<\gamma \leq 1
\end{aligned}
$$

$$
\begin{aligned}
& \frac{1}{L^{\gamma} \Gamma(3-\gamma)} \sum_{j=0}^{k}\left(U_{j+1}-2 U_{j}+U_{j-1}\right) \\
& \quad \times\left((k-j+1)^{2-\gamma}-(k-j)^{2-\gamma}\right) \\
& \quad+\eta \int_{0}^{s_{k+1}} K\left(s_{k+1}, s\right) u_{n}(s) d s=g\left(s_{k+1}\right), \\
& 0<\gamma \leq 1, \\
& \frac{1}{L^{\gamma} \Gamma(4-\gamma)} \sum_{j=0}^{k}\left(U_{j+2}-3 U_{j+1}+3 U_{j}\right. \\
& \left.-U_{j-1}\right)\left((k-j+1)^{3-\gamma}-(k-j)^{3-\gamma}\right) \\
& +\eta \int_{0}^{s_{k+1}} K\left(s_{k+1}, s\right) u_{n}(s) d s=g\left(s_{k+1}\right), \\
& 2<\gamma \leq 3,
\end{aligned}
$$

where $U_{0}:=u(0), U_{1}:=\frac{d}{d t} u(0)-L \frac{d^{2}}{d t^{2}} u(0)$ and $U_{-1}:=$ $u(0)-\frac{d}{d t} u(0)$ are known initial conditions.

Otherwise, for $\quad \int_{0}^{s_{k+1}} K\left(s_{k+1}, s\right) u_{n}(s) d s$, we have

$$
\begin{aligned}
\int_{0}^{s_{k+1}} K\left(s_{k+1}, s\right) u_{n}(s) d s & =\int_{0}^{s_{k+1}} K\left(s_{k+1}, s\right) \sum_{i=0}^{n} U_{i} S_{i}(s) d s \\
& =\sum_{i=0}^{n} U_{i} \int_{0}^{s_{k+1}} K\left(s_{k+1}, s\right) S_{i}(s) d s
\end{aligned}
$$

Let

$$
W_{i}=\int_{0}^{s_{k+1}} K\left(s_{k+1}, s\right) S_{i}(s) d s, i=0,1, \ldots, n .
$$

Hence $\int_{0}^{s_{k+1}} K\left(s_{k+1}, s\right) u_{n}(s) d s=\sum_{i=0}^{k+1} U_{i} W_{i},\left(W_{i}=0\right.$, for $i>k+1)$. Now, using the boundary condition, we may calculate $U_{1}, U_{2}, \ldots, U_{n}$ by the obtained recursive equations (17)-(19) and thus for Eq. (1), we may achieve the approximation $u(t)=u_{n}(t)$.

An algorithm for approximation of FIDEs by TTM is presented as Algorithm 1.

Algorithm 1. An algorithm to approximate solution of high-order FIDEs via TTM.

Stage 1. Input $g(t), K(t, s), n$ and $b$.

Stage 2. Put $L \leftarrow \frac{b}{n}$.

Stage 3. For $k=0,1,2,3, \ldots, n$, set $s_{k} \leftarrow k L$.

Stage 4. For $k=0,1,2,3, \ldots, n$, select TBFs $S_{k}(t)$.

Stage 5. For $k=0,1,2,3, \ldots, n-1$ and $i=0,1,2,3, \ldots, k+1$, assume

$$
W_{i}=\int_{0}^{s_{k+1}} K\left(s_{k+1}, s\right) S_{i}(s) d s .
$$


Stage 6. Put

(a) for $m=1$ :

$$
\begin{array}{r}
\frac{1}{L^{\gamma} \Gamma(2-\gamma)} \sum_{j=0}^{k}\left(U_{j+1}-U_{j}\right) \\
\left((k-j+1)^{1-\gamma}-(k-j)^{1-\gamma}\right) \\
+\eta \sum_{i=0}^{k+1} U_{i} W_{i}=g\left(s_{k+1}\right),
\end{array}
$$

where $U_{0}=u(0)$.

(b) For $m=2$ :

$$
\begin{aligned}
& \frac{1}{L^{\gamma} \Gamma(3-\gamma)} \sum_{j=0}^{k}\left(U_{j+1}-2 U_{j}+U_{j-1}\right) \\
& \left((k-j+1)^{2-\gamma}-(k-j)^{2-\gamma}\right) \\
& +\eta \sum_{i=0}^{k+1} U_{i} W_{i}=g\left(s_{k+1}\right)
\end{aligned}
$$

where $U_{0}=u(0)$ and $U_{-1}=u(0)-L \frac{d u(0)}{d t}$. c) For $m=3$ :

$$
\begin{aligned}
& \frac{1}{L^{2} \Gamma(4-\gamma)} \sum_{j=0}^{k}\left(U_{j+2}-3 U_{j+1}+3 U_{j}-U_{j-1}\right) \\
& \quad \times\left((k-j+1)^{3-\gamma}-(k-j)^{3-\gamma}\right) \\
& \quad+\eta \sum_{i=0}^{k+1} U_{i} W_{i}=g\left(s_{k+1}\right),
\end{aligned}
$$

where $U_{0}=u(0), U_{1}=\frac{d}{d t} u(0)-L \frac{d^{2} u(0)}{d t^{2}}$ and $U_{-1}=u(0)-L \frac{d u(0)}{d t}$.

Stage 7. For $p=1,2,3, \ldots, n$, from an equation of degree one, calculate every $U_{p}$.

Stage 8. The approximate solution for the FIDEs is

$$
u_{n}(t)=\sum_{k=0}^{n} U_{k} S_{k}(t)
$$

\section{Examples}

Various examples in this section are now provided to get familiar with the TTM for high-order FDIEs. The software Mathematicall has been utilized in these examples for computations and graphs.

Example 1 We offer the Volterra integro-differential equation for the first example:

$$
\begin{aligned}
D^{\gamma} u(t)-\int_{0}^{t}(t+s) u(s) d s & =1-\frac{(2 \gamma+3) t^{\gamma+2}}{\Gamma(\gamma+3)}, \\
0 & \leq t \leq 1,0 \leq \gamma \leq 1,
\end{aligned}
$$

with the exact solution $u(t)=\frac{t^{\gamma}}{\Gamma(\gamma+1)}$ and the initial condition

$$
u(0)=0 .
$$

Conforming to what was mentioned in section 3, utilizing TTM for Eq. (22), we may calculate $U_{1}, U_{2}, \ldots, U_{n}$ and then gain the approximate solution $u_{n}(t)$ for Eq. (22).

The approximate solutions for $\gamma=0.9$ and $n=50$ obtained via various values of $t$ in table 1 are illustrated. We may see the exact and approximate solution via $\gamma=0.9$ and $n=50$, in figure 1 .

Test example 1 with details is illustrated in Appendix I.

Example 2 We offer the Volterra integro-differential equation for the second example:

$$
\begin{aligned}
D^{\gamma} u(t)-\int_{0}^{t} u(t) d t & =\frac{4^{\gamma} t^{\gamma}}{\sqrt{\pi}}-\frac{t^{2 \gamma+1}}{2 \Gamma\left(\gamma+\frac{3}{2}\right)}, \\
0 & \leq t \leq 1,1 \leq \gamma \leq 2,
\end{aligned}
$$

via the exact solution $u(t)=\frac{t^{2 \gamma}}{\Gamma\left(\gamma+\frac{1}{2}\right)}$ and the initial conditions

$$
u(0)=0, \frac{d u(0)}{d t}=0 .
$$

Table 1. Comparative outcomes of Example 1.

\begin{tabular}{lllc}
\hline$t$ & TTM & Exact & Absolute error \\
\hline 0.0 & 0.0 & 0.0 & 0.0 \\
0.2 & 0.242498 & 0.244263 & $3.85405 \times 10^{-3}$ \\
0.4 & 0.44815 & 0.455811 & $7.66069 \times 10^{-3}$ \\
0.6 & 0.642711 & 0.656548 & $13.837 \times 10^{-3}$ \\
0.8 & 0.844837 & 0.850573 & $5.73563 \times 10^{-3}$ \\
1.0 & 1.07128 & 1.03975 & $31.5248 \times 10^{-3}$ \\
\hline
\end{tabular}

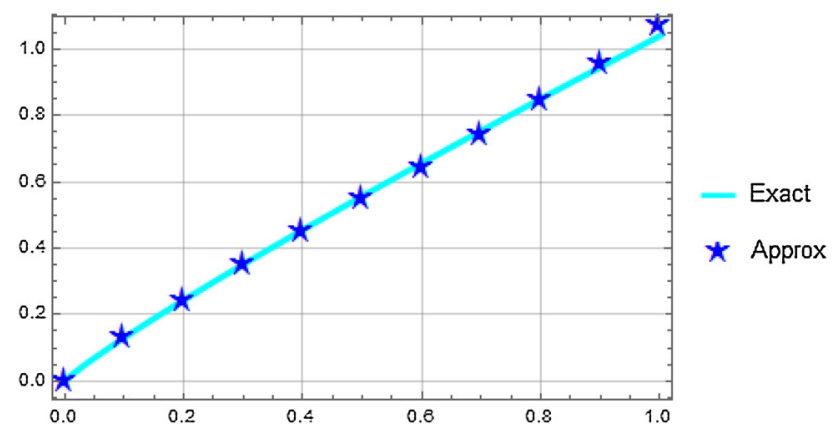

Figure 1. Agreement by TTM for Eq. (22) and exact solution. 


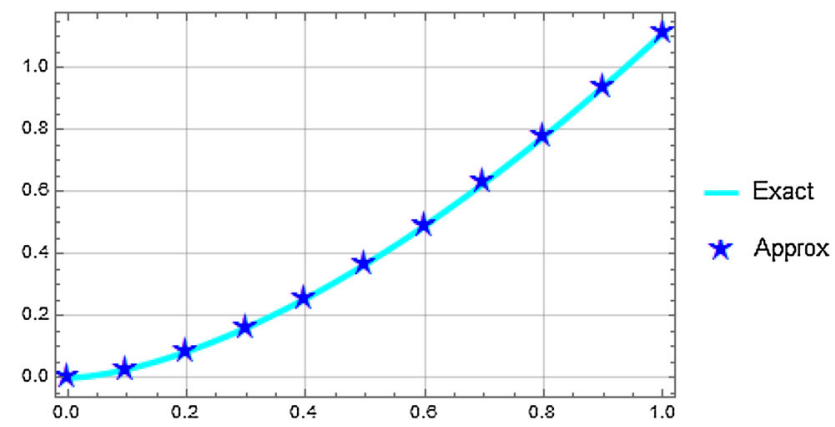

Figure 2. Agreement by TTM for Eq. (24) and exact solution.

Table 2. Comparative outcomes of Example 2.

\begin{tabular}{lllc}
\hline$t$ & TTM & Exact & Absolute error \\
\hline 0.0 & 3.0 & 3.0 & 0.0 \\
0.2 & 2.99983 & 2.99983 & $3.04308 \times 10^{-6}$ \\
0.4 & 2.99299 & 2.99295 & $35.2995 \times 10^{-6}$ \\
0.6 & 2.94082 & 2.94063 & $191.503 \times 10^{-6}$ \\
0.8 & 2.79051 & 2.79012 & $386.906 \times 10^{-6}$ \\
1.0 & 2.99898 & 3.0 & $1.02379 \times 10^{-6}$ \\
\hline
\end{tabular}

The unknown coefficients $U_{i}, i=1,2, \ldots, n$ for Eq. (24) are determined by the TTM based on section 3 .

In figure 2 and in table 2, the exact and approximate solutions featuring $\gamma=0.8$ and $n=500$ through applying TTM can be seen. With the knowledge that $\gamma=0.8$ and $n=500$, the approximate solution obtained by the TTM matches the exact solution.

Example 3 We offer the Volterra integro-differential equation for the third example:

$$
\begin{gathered}
D^{\gamma} u(t)+\int_{0}^{t} t u(t) d t=\frac{t^{3 \gamma+2}}{3 \gamma+1}-\frac{t^{6 \gamma+2}}{6 \gamma+1} \\
-\frac{3 \gamma \Gamma(3 \gamma) t^{2 \gamma}}{\Gamma(2 \gamma+1)}+\frac{6 \gamma \Gamma(6 \gamma) t^{5 \gamma}}{\Gamma(5 \gamma+1)}-3 t^{2} \\
0 \leq t \leq 1, \quad 1 \leq \gamma \leq 2
\end{gathered}
$$

including the following initial conditions and the exact solution $u(t)=3-t^{3 \gamma}+t^{6 \gamma}$ :

$$
u(0)=3, \quad \frac{d u(0)}{d t}=0 .
$$

In figure 3 and in table 3, the exact and approximate answers featuring $\gamma=1.8$ and $n=500$ through applying TTM, can be seen. For $\gamma=1.8$ and $n=500$, the solution that we have gained is in match with the exact solution.

Example 4 We offer the Volterra integro-differential equation, for the fourth example:

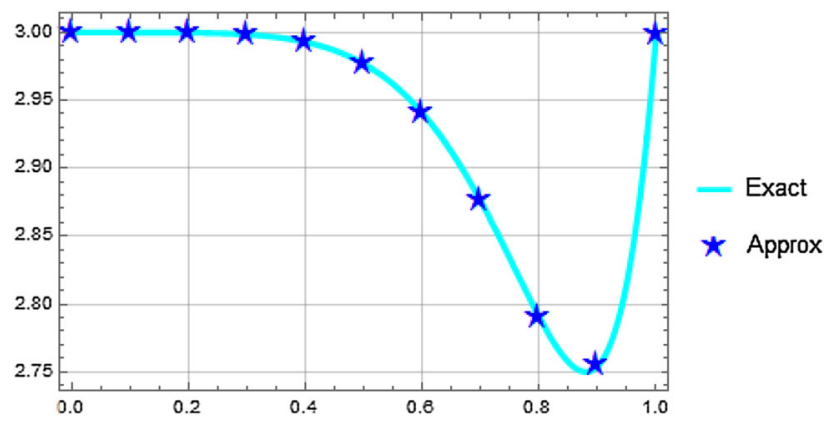

Figure 3. Agreement by TTM for Eq. (26) and exact solution.

Table 3. Comparative outcomes of Example 3.

\begin{tabular}{lllc}
\hline$t$ & TTM & Exact & Absolute error \\
\hline 0.0 & 3.0 & 3.0 & 0.0 \\
0.2 & 2.99983 & 2.99983 & $3.04308 \times 10^{-6}$ \\
0.4 & 2.99299 & 2.99295 & $35.2995 \times 10^{-6}$ \\
0.6 & 2.94082 & 2.94063 & $191.503 \times 10^{-6}$ \\
0.8 & 2.79051 & 2.79012 & $386.906 \times 10^{-6}$ \\
1.0 & 2.99898 & 3.0 & $1.02379 \times 10^{-3}$ \\
\hline
\end{tabular}

$$
\begin{aligned}
D^{\gamma} u(t)-\int_{0}^{t} u(t) d t= & \frac{3 t\left(t^{3}\right)^{\gamma}}{6 \gamma+2} \\
& -\frac{2 t^{\frac{3 \gamma}{2}+1}}{3 \gamma+2}+\frac{3 \Gamma\left(\frac{3 \gamma}{2}\right) t^{\gamma / 2}}{\Gamma\left(\frac{\gamma}{2}\right)}-\frac{9 \Gamma(3 \gamma) t^{2 \gamma}}{4 \Gamma(2 \gamma)}-t \\
& 0 \leq t \leq 1,2 \leq \gamma \leq 3
\end{aligned}
$$

although the initial conditions:

$$
u(0)=1, \quad \frac{d u(0)}{d t}=0, \quad \frac{d^{2} u(0)}{d t^{2}}=0 .
$$

The unknown coefficients $U_{i}, i=1,2, \ldots, n$ with the TTM, matching to section 3 for Eq. (28) are determined. The approximate solutions for $\gamma=2.3$ and $n=50$ obtained via various values of $t$ in table 4 are illustrated.

In figure 4 , we can confirm the correspondence between the exact answer and the approximate solution with $\gamma=2.3$.

For $\gamma=2.3$, the exact solution with $u(t)=1-\frac{3 t^{3 \gamma}}{2}+t^{\frac{3 y}{2}}$ and the obtained approximate solution are consistent.

Example 5 We offer the Volterra integro-differential equation for the fifth example:

$$
D^{\gamma} u(t)-\int_{0}^{t} u(s) d s=18 t-\frac{t^{2}}{2}+t^{3}-\frac{3 t^{4}}{4}-6
$$


Table 4. Comparative outcomes of Example 4.

\begin{tabular}{lllc}
\hline$t$ & TTM & Exact & Absolute error \\
\hline 0.0 & 1.0 & 1.0 & 0.0 \\
0.2 & 1.00382 & 1.00385 & $36.0925 \times 10^{-6}$ \\
0.4 & 1.0395 & 1.03968 & $185.174 \times 10^{-6}$ \\
0.6 & 1.12715 & 1.12745 & $298.121 \times 10^{-6}$ \\
0.8 & 1.14206 & 1.14141 & $641.586 \times 10^{-6}$ \\
1.0 & 0.504706 & 0.5 & $4.70572 \times 10^{-3}$ \\
\hline
\end{tabular}

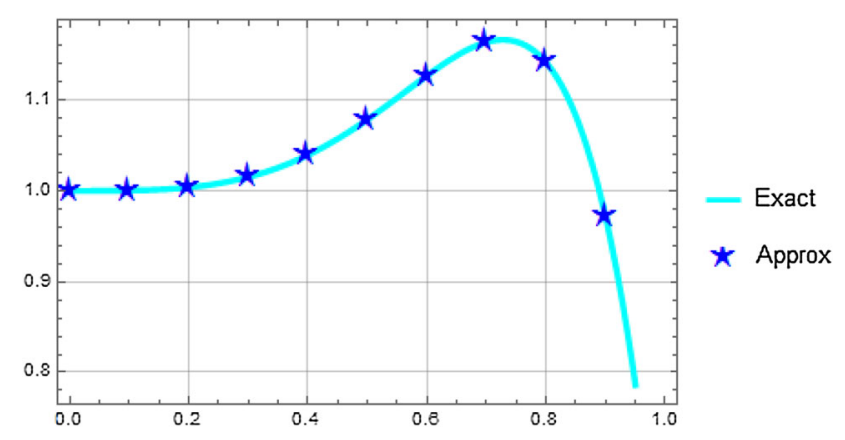

Figure 4. Agreement by TTM for Eq. (28) and exact solution.

$$
1 \leq \gamma \leq 2,0 \leq t \leq 1,
$$

with the following initial conditions and the exact solution $u(t)=3 t^{3}-3 t^{2}+t$ :

$$
u(0)=-6, \quad \frac{d u(0)}{d t}=1 .
$$

Table 5 shows the approximate solution determined for various values of $\gamma, n=500$ and various values of $t$.

In figure 5, we confirm the correspondence between the exact answer and the approximate solution with $\gamma=$ $1.1,1.5,1.9$ by TTM for $n=500$.

Table 5. Comparative outcomes of Example 5 with various values of $\gamma$ and $n=500$.

\section{TTM}

\begin{tabular}{lllcl}
\hline$t$ & $\gamma=1.50$ & $\gamma=1.70$ & $\gamma=1.90$ & Exact \\
\hline 0.0 & 0.00 & 0.00 & 0.00 & 0.00 \\
0.2 & 0.156927 & 0.241038 & 0.319699 & 0.32 \\
0.4 & 0.567237 & 0.58592 & 0.639287 & 0.64 \\
0.6 & 1.60812 & 1.37563 & 1.19871 & 1.2 \\
0.8 & 3.52252 & 2.87066 & 2.23804 & 2.24 \\
1.0 & 6.51626 & 5.30314 & 3.99825 & 4.0 \\
\hline
\end{tabular}

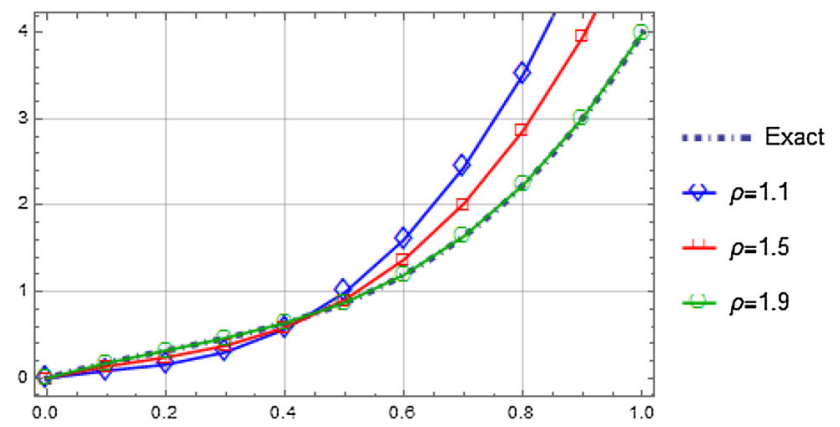

Figure 5. Comparative outcomes via TTM of Example 5 for various values of $t, n=500$ and $\gamma$.

\section{Conclusion}

We have prosperously utilized TTM to acquire approximate solutions of the FIDEs. In this paper, initially various reallife phenomena related to FIDEs are introduced. Following this, discretization of the fractional derivative for different orders and the TBFs, lemmas and theorems are presented in section 2, to approximate the function of the solution, including convergence of approximate solution function with TBFs. In section 3, via discretization of the fractional derivative, the approximate solution of FIDEs is determined from a linear combination of BFs with trigonometric transforms.

The results demonstrate that a few iterations of TTM will result in useful approximate solutions.

The suggested technique can be utilized for solving fractional integral equations FIEs, fractional integro-partial differential equations FIPDEs, FDEs, fractional partial differential equations $F P D E s$, fractional differential system equations $F D S E s$ and fractional partial differential system equations FPDSEs.

\section{Appendix I. Illustration of Example 1 with details}

Consider the fractional Volterra integro-differential equation of example 1:

$$
\begin{gathered}
D^{\gamma} u(t)-\int_{0}^{t}(t+s) u(s) d s=1-\frac{(2 \gamma+3) t^{\gamma+2}}{\Gamma(\gamma+3)}, \\
0 \leq t \leq 1,0 \leq \gamma \leq 1
\end{gathered}
$$

with the exact solution $u(t)=\frac{t^{\gamma}}{\Gamma(\gamma+1)}$ and the initial condition

$$
u(0)=0 .
$$

Algorithm 1 is implemented as follows. 
Stage 1. Input $g(t)=1-\frac{(2 \gamma+3) t^{\gamma+2}}{\Gamma(\gamma+3)}, K(t, s)=t+s, n=50$ and $b=1$.

Stage 2. Put $L \leftarrow \frac{1}{50}$.

Stage 3. Set $s_{k} \leftarrow \frac{k}{50}, \quad k=0,1,2,3, \ldots, 50$.

Stage 4. From Eq. (7)

$$
\begin{gathered}
S_{0}(t)=\left\{\begin{array}{cc}
0.5(1+\cos (50 \pi)), & 0 \leq t \leq \frac{1}{50}, \\
0, & \text { otherwise, }
\end{array}\right. \\
S_{k}(t)=\left\{\begin{array}{cc}
0.5\left(1+\cos \left(50 \pi\left(t-s_{k}\right)\right)\right), & s_{k-1} \leq t \leq s_{k}, \\
0.5\left(1+\cos \left(50 \pi\left(t-s_{k}\right)\right)\right), & s_{k} \leq t \leq s_{k+1}, \\
0, & \text { otherwise, }
\end{array}\right. \\
S_{50}(t)=\left\{\begin{array}{cc}
0.5(1+\cos (50 \pi(t-1))), & \frac{1}{50} \leq t \leq 1, \\
0, & \text { otherwise, }
\end{array}\right.
\end{gathered}
$$

where $k=2,3,4, \ldots, 49$.

Stage 5. Consider

$$
W_{i}=\int_{0}^{s_{k+1}}\left(s_{k+1}+s\right) S_{i}(s) d s
$$

for $k=0,1, \ldots, 49$ and $i=0,1, \ldots, k+1$.

Stage 6. Construct recursive equations

$$
\begin{aligned}
& \frac{1}{\left(\frac{1}{50}\right)^{\gamma} \Gamma(2-\gamma)} \sum_{j=0}^{k}\left(U_{j+1}-U_{j}\right) \\
& \times\left((k-j+1)^{1-\gamma}-(k-j)^{1-\gamma}\right) \\
& +\eta \sum_{i=0}^{k+1} U_{i} W_{i}=1-\frac{(2 \gamma+3)\left(s_{k+1}\right)^{\gamma+2}}{\Gamma(\gamma+3)} .
\end{aligned}
$$

where $U_{0}=u(0)=0$.

Stage 7. For $p=0$, we have

$$
\begin{gathered}
\frac{1}{\left(\frac{1}{50}\right)^{\gamma} \Gamma(2-\gamma)}\left(U_{1}-U_{0}\right)+\eta U_{0} W_{0} \\
=1-\frac{(2 \gamma+3)\left(s_{1}\right)^{\gamma+2}}{\Gamma(\gamma+3)}
\end{gathered}
$$

Hence we may calculate $U_{1}$. For $p=2, \ldots, n$, we may calculate every $U_{k}$ by solving an equation of degree one.

Stage 8. The approximate solution is

$$
u_{50}(t)=U_{0} S_{0}(t)+U_{1} S_{1}(t)+\cdots+U_{50} S_{50}(t) .
$$

\section{References}

[1] Sun H, Zhang Y, Baleanu D, Chen W and Chen Y 2018 A new collection of real world applications of fractional calculus in science and engineering. Commun. Nonlinear Sci. Numer. Simul. 64(2): 213-231
[2] Baleanu D and Luo A C 2014 In: Machado J T (Ed.) Discontinuity and complexity in nonlinear physical systems. Springer

[3] Kilbas A A, Srivastava H M and Trujillo J J 2006 Theory and application of fractional differential equations. Amsterdam: Elsevier

[4] Podlubny I 1998 An introduction to fractional derivatives, fractional differential equations, to methods of their solution and some of their applications. In: Fractional differential equations, vol. 198. Academic Press

[5] Kythe P and Puri P 2011 Computational methods for linear integral equations. Berlin: Springer

[6] Bagley R L and Torvik P J 1983 A theoretical basis for the application of fractional calculus to viscoelasticity. J. Rheol. 27(3): 201-210

[7] Gushing J M 2010 Volterra integro-differential equations in population dynamics. In: Mathematics of biology. BerlinHeidelberg: Springer

[8] Baleanu D, Agheli B, Adabitabar Firozja M and Mohamed Al Qurashi M 2017 A method for solving nonlinear Volterra's population growth model of noninteger order. $A d v$. Differ. Equ. 2017: 368

[9] Agrawal O P 2004 A general formulation and solution scheme for fractional optimal control problems. Nonlinear Dyn. 38(1-4): 323-337

[10] Rabiei K, Ordokhani Y and Babolian E 2018 Fractionalorder Legendre functions and their application to solve fractional optimal control of systems described by integrodifferential equations. Acta Appl. Math. 158(1): 87-106

[11] Baleanu D, Darzi R and Agheli B 2017 New study of weakly singular kernel fractional fourth-order partial integro-differential equations based on the optimum q-homotopic analysis method. J. Comput. Appl. Math. 320: 193-201

[12] Abbasbandy S, Hashemi M S and Hashim I 2013 On convergence of homotopy analysis method and its application to fractional integro-differential equations. Quaest. Math. 36(1): 93-105

[13] Nawaz Y 2011 Variational iteration method and homotopy perturbation method for fourth-order fractional integro-differential equations. Comput. Math. Appl. 61(8): 2330-2341

[14] Sayevand K, Fardi M, Moradi E and Boroujeni F H 2013 Convergence analysis of homotopy perturbation method for Volterra integro-differential equations of fractional order. Alex. Eng. J. 52(4): 807-812

[15] Amer S M, Saleh M H, Mohamed M A and Abdelrhman N S 2013 Variational iteration method and Adomian decomposition method for fourth-order fractional integro-differential equations. Int. J. Comput. Appl. 80(6): 7-14

[16] Yuzbasi S 2016 A collocation method based on Bernstein polynomials to solve nonlinear Fredholm-Volterra integrodifferential equations. Appl. Math. Comput. 273: 142-154

[17] Eslahchi M R, Dehghan M and Parvizi M 2014 Application of the collocation method for solving nonlinear fractional integro-differential equations. J. Comput. Appl. Math. 257: $105-128$

[18] Korvenpaa J, Kuusi T and Palatucci G 2016 Fractional superharmonic functions and the Perron method for nonlinear integro-differential equations. Math. Ann. 369(3-4): 1443-1489

[19] Yi M, Wang L and Huang J 2016 Legendre wavelets method for the numerical solution of fractional integro-differential 
equations with weakly singular kernel. Appl. Math. Model. 40(4): 3422-3437

[20] Perfilieva I 2006 Fuzzy transforms: theory and applications. Fuzzy Sets Syst. 157(8): 993-1023

[21] Khastan A, Perfilieva I and Alijani Z 2016 A new fuzzy approximation method to Cauchy problems by fuzzy transform. Fuzzy Sets Syst. 288: 75-95

[22] Khastan A, Alijani Z and Perfilieva I 2016 Fuzzy transform to approximate solution of two-point boundary value problems. Math. Methods Appl. Sci.https://doi.org/10.1002/mma.3832
[23] Tomasiello S 2016 An alternative use of fuzzy transform with application to a class of delay differential equations. Int. J. Comput. Math. 94(9): 1719-1726

[24] Chen W and Shen Y 2014 Approximate solution for a class of second-order ordinary differential equations by the fuzzy transform. J. Intell. Fuzzy Syst. 27(1): 73-82

[25] Li C, Zhao Z and Chen Y 2011 Numerical approximation of nonlinear fractional differential equations with sub-diffusion and super-diffusion. Comput. Math. Appl. 62(3): 855-875 